\title{
Results from Tunka-133 (5 years observation) and from the Tunka-HiSCORE prototype
}

\author{
V.V. Prosin ${ }^{1, a}$, S.F. Berezhnev ${ }^{1}$, N.M. Budnev² ${ }^{2}$ M. Brückner ${ }^{3}$, A. Chiavassa ${ }^{4}$,
} O.A. Chvalaev ${ }^{2}$ A.V. Dyachok ${ }^{2}$, S.N. Epimakhov ${ }^{5}$, A.V. Gafarov², O.A. Gress², T.I. Gress ${ }^{2}$, D. Horns ${ }^{5}$, N.N. Kalmykov ${ }^{1}$, N.I. Karpov ${ }^{1}$, S.N. Kiryuhin ${ }^{2}$, E.N. Konstantinov ${ }^{2}$, A.V. Korobchenko ${ }^{2}$, E.E. Korosteleva ${ }^{1}$, V.A. Kozhin ${ }^{1}$, M. Kunnas ${ }^{5}$, L.A. Kuzmichev ${ }^{1,2}$, B.K. Lubsandorzhiev ${ }^{6}$, N.B. Lubsandorzhiev ${ }^{1}$, R.R. Mirgazov ${ }^{2}$, R.D. Monkhoev ${ }^{2}$, R. Nachtigall ${ }^{5}$, E.A. Osipova ${ }^{1}$, A.L. Pakhorukov' ${ }^{2}$, M.I. Panasyuk ${ }^{1}$, L.V. Pankov ${ }^{2}$, V.A. Poleschuk ${ }^{2}$, E.G. Popova ${ }^{1}$, A. Porelli ${ }^{7}$, V.S. Ptuskin ${ }^{8}$, G.I. Rubtsov ${ }^{6}$, M. Rüger ${ }^{7}$, V.S. Samoliga ${ }^{2}$, Y.A. Semeney ${ }^{2}$, A.A. Silaev ${ }^{1}$, A.A. Silaev (jun. $)^{1}$, A.V. Skurikhin ${ }^{1}$, C. Spiering ${ }^{7}$, L.G. Sveshnikova ${ }^{1}$, M. Tluczykont ${ }^{5}$, R. Wischnewski ${ }^{7}$, A.V. Zagorodnikov ${ }^{2}$, and A.V. Zurbanov ${ }^{2}$

${ }^{1}$ Skobeltsyn Institute of Nuclear Physics, Lomonosov Moscow State University, Moscow, Russian Federation

${ }^{2}$ Institute of Applied Physics, Irkutsk State University, Irkutsk, Russian Federation

${ }^{3}$ Institute für Informatik, Humboldt University, Berlin, Germany

${ }^{4}$ University of Turin, Turin, Italy

${ }^{5}$ Institute fur Experimentalphysik, University of Hamburg, Hamburg, Germany

${ }^{6}$ Institute of Nuclear Research, Russian Academy of Sciences, Moscow, Russian Federation

${ }^{7}$ DESY Zeuthen, Germany

${ }^{8}$ Pushkov Institute of Terrestrial Magnetism, Ionosphere and Radio Waves Distribution, Russian Academy of Sciences, Moscow, Russian Federation

\begin{abstract}
Data obtained with two detectors located at the Tunka Cosmic Ray facility are presented. The Cherenkov light array for registration of extensive air showers (EAS) Tunka-133 collected data during 5 winter seasons since 2009 to 2014. The differential energy spectrum of all particles and the dependence of the average maximum depth on the energy in the range of $6 \cdot 10^{15}-10^{18} \mathrm{eV}$ measured for 1540 hours of observation are presented.

The preliminary all particle energy spectrum by the data of TunkaHiSCORE prototype array, installed in 2013, is presented. Some additional experiments in the Tunka Valley are briefly described.
\end{abstract}

\section{Introduction}

EAS Cherenkov light array Tunka-133 with $\sim 3 \mathrm{~km}^{2}$ geometric area operated since 2009 [1]. Five winter seasons of data acquisition $\left(\sim 10^{7}\right.$ triggers $)$ and high quality of information permitted us to reconstruct primary energy spectrum and mass composition in the energy range $6 \cdot 10^{15}-10^{18} \mathrm{eV}$. This energy range is the most important for understanding of transition from Galactic to extragalactic cosmic rays (CR).

\footnotetext{
a e-mail: v-prosin@yandex.ru
} 
To expand the measurement range to lower energies the new array Tunka-HiSCORE [2] data has been used. The HiSCORE concept has been described in [3] and [4]. The preliminary primary energy spectrum in the range $200 \mathrm{TeV}-20 \mathrm{PeV}$ by the data of the first season of 9 HiSCORE stations operation is presented. Tunka-HiSCORE stations are located at the same area as Tunka-133 detectors. In 2013 the 9 station array, arranged on a rectangular grid, covered an area of $0.09 \mathrm{~km}^{2}$, which will be increased to about $0.25 \mathrm{~km}^{2}$ in 2014 .

\section{The experiments development In Tunka Valley}

Some additional experiments are carried out in the Tunka Valley astrophysical site.

The scintillation detectors used for some years at KASCADE-Grande array [5] has been transported to the Tunka Valley in 2013. These detectors were originally designed for the EAS-TOP array [6] in Italy. The area of each detector is $0.64 \mathrm{~m}^{2}$. They are planned to be grouped into 19 stations located near the centers of Tunka-133 clusters. In each station 12 detectors will be located on the ground surface and 8 detectors - underground for registration of muons.

The Tunka radio extension (Tunka-REX) [7] experiment is operating together with Tunka-133.

The main purpose of Tunka-HiSCORE is to select high energy gamma quanta from point like sources. To solve this task it is planned to reach an accuracy of EAS arrival direction measurement better than $0.1^{\circ}$.

It is planned to install several IACT telescopes [2] for common operation with TunkaHiSCORE.

\section{Data processing}

The Tunka-133 data were processed using the original codes, in which all fitting and conversion functions were obtained from the analysis of events simulated by CORSIKA for the energy range of $10^{15}-10^{18} \mathrm{eV}$. The details were described in [8].

Shower arrival direction, characterized by zenith and azimuth angles is reconstructed by fitting the measured delays with curve shower front: $T_{i}-T_{f}=R \cdot(R+500) /(c \cdot F)$, where $T_{f}$ - estimated delay for a plane front, $R$ - the perpendicular distance from the shower axis in meters $c$ - the speed of light, $F$ is a parameter. This approximate formula is derived from the analysis of CORSIKA simulated showers. The formula, on the one hand, has a non-zero value of the derivative at $R=0$ (conical shape, typical for Cherenkov radiation at short distances from the axis) and on the other hand, has only one shape parameter which is essential for processing of relatively small showers.

EAS maximum depth is derived from two parameters - the steepness of the amplitudedistance function (ADF) and the pulse width at a core distance of $400 \mathrm{~m} \tau(400)$ [8].

Data for 262 clear moonless nights of observation was collected during 5 winter seasons of 2009-2014. Trigger condition was a coincidence of pulses in 3 detectors of a cluster inside the time gate of $0.5 \mu \mathrm{s}$. It was accumulated about $10^{7}$ trigger events for 1540 hours during these nights.

Tunka-HiSCORE data presented here have been collected during $80 \mathrm{~h}$ for 13 clean moonless nights from February 21st to April, 3th, 2014. All the 9 stations recorded about $1.5 \cdot 10^{7}$ Cherenkov light pulses. The pulses treated to belong to the same shower if the time difference among them was less than $2 \mu$ s. It was selected about $1.7 \cdot 10^{6}$ showers with 4 and more hitted stations. The showers selected were processed with algorithms similar to those used for Tunka-133 [8]. 


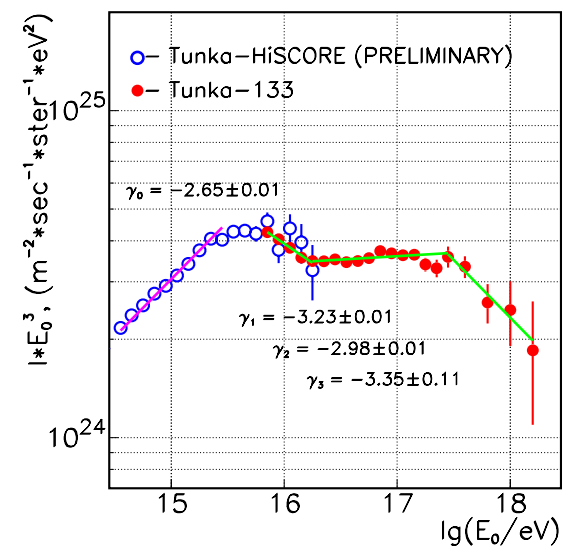

Figure 1. Differential primary cosmic rays (CR) energy spectrum.

\section{Energy spectrum}

To construct the Tunka-133 spectrum the events were selected with zenith angles $\theta \leq 45^{\circ}$ and the core position inside a circle of radius $R_{c} \leq 450 \mathrm{~m}$ for energy $E_{0}<5 \cdot 10^{16} \mathrm{eV}$ and a circle of radius $R_{c}<800 \mathrm{~m}$ for showers with energy $E_{0} \geq 5 \cdot 10^{16} \mathrm{eV}$. Comparison of the spectra for these two effective areas showed that starting from the above mentioned energy spectra within the error bars are the same, but the event statistics in the second round is 3 times more which is essential for energies $E_{0} \geq 10^{17} \mathrm{eV}$.

Efficiency of shower selection inside the circle with $R_{c} \leq 450 \mathrm{~m}$ reaches $100 \%$ for energies $E_{0} \geq 6 \cdot 10^{15} \mathrm{eV}$. Total number of events above this energy is 270.000 . About 3.000 events, selected in the circle with $R_{c}<800 \mathrm{~m}$ have $E_{0} \geq 10^{17} \mathrm{eV}$.

The events for construction of the preliminary Tunka-HiSCORE spectrum were selected from events recorded inside the zenith angle $\theta \leq 25^{\circ}$ and the effective area consisting of four circles of radius $75 \mathrm{~m}$ around the centers of 4 squares arranged by 9 stations. The number of events with energy $E_{0} \geq 3 \cdot 10^{14} \mathrm{eV}$ (for which the efficiency is close to $100 \%$ ) is about 140.000 .

The resulting differential Tunka-133 energy spectrum is shown in Fig. 1 together with the preliminary spectrum of Tunka-HiSCORE. One can see that Tunka-HiSCORE can provide information on the detailed shape of the spectrum at and before the knee. But more statistics is needed for this purpose. The spectrum of Tunka-133 shows a number of features - deviations from the power law. At the energy of about $2 \cdot 10^{16} \mathrm{eV}$ the power law index changes from $\gamma=3.23 \pm 0.01$ to $\gamma=2.98 \pm 0.01$. Points of the spectrum are consistent with such an index till the energy $E_{0}=3 \cdot 10^{17} \mathrm{eV}$. Above this energy spectrum becomes steeper with $\gamma=3.35 \pm 0.11$ (the second "knee"). There is a possibility of more complex description of the second "knee" with a small intermediate increase of the absolute value of the index to $\gamma=3.06 \pm 0.03$ at the energy of $8 \cdot 10^{16} \mathrm{eV}$.

In Fig. 2 the spectrum is compared with a number of other experimental data. The spectra of all the experiments shown in Fig. 2: KASCADE [10], EAS-TOP [6], Tibet [11], HEGRA [12] - are practically indistinguishable at the energy of the first (classical) knee.

At energies below the knee there is an agreement between the preliminary result of TunkaHiSCORE on the one hand and results of HEGRA [12] and Tibet [11] on the other hand.

There is an agreement between the result of Tunka-133 and the spectra of GAMMA [9], KASCADE-Grande [5] and Ice-TOP [13] in the intermediate energy range $10^{16}-10^{17} \mathrm{eV}$. Noticeable in Fig. 2 difference among the spectra at $E_{0}$ about $10^{17} \mathrm{eV}$ can be eliminated by 


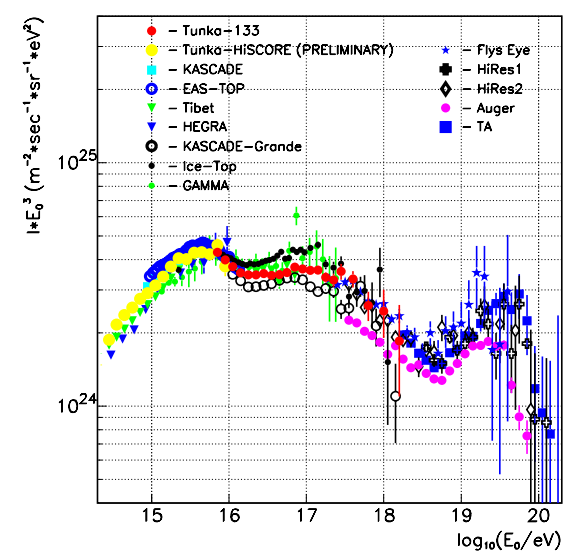

Figure 2. Comparison of energy spectra obtained at Tunka with some other experimental results.

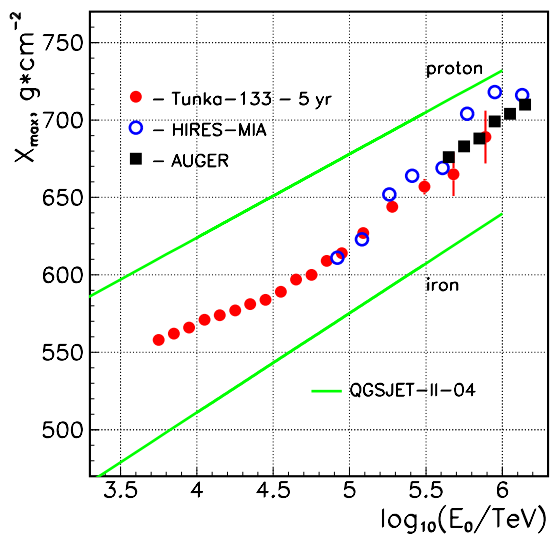

Figure 3. Mean Depth of Maximum vs. Energy.

correcting energy by $3 \%$ only. Such energy shift is much smaller than the absolute accuracy of these experiments.

For highest energies Tunka-133 data are in agreement with Fly's Eye [14], HiRes [15] and Telescope Array (TA) [16] data, and also (to less extend) with Auger [17].

\section{The depth of maximum}

The methods of EAS maximum depth $X_{\max }$ measurement are described in [8]. Events for this analysis are selected in a circle of radius $450 \mathrm{~m}$. To get a uniform estimation for $b_{A}$ over a wide range of energies, we remove from the analysis detectors at distances larger than $250 \mathrm{~m}$ from the core during the last step of parameters reconstruction. The experimental dependence of mean $\left\langle X_{\max }\right\rangle$ vs. primary energy $E_{0}$ in the energy range of $6 \cdot 10^{15}-10^{18} \mathrm{eV}$ is presented in Fig. 3. The experimental points are compared with the points of HiRes-MIA experiment [15] and fluorescent light detectors of Pierre Auger Observatory [17]. One can see an agreement of Cerenkov light Tunka experiment results with direct fluorescent light observations. The new measurements are compared with the theoretical curves simulated with the QGSJET-II-04 model for primary protons and iron nuclei. 


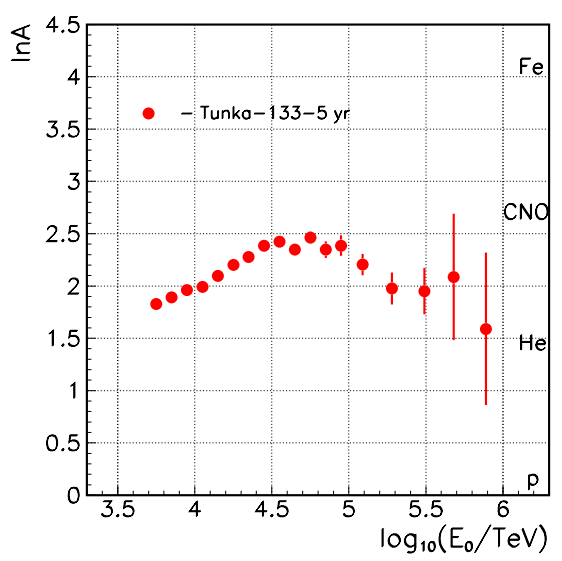

Figure 4. Mean Logarithmic Mass vs. Energy.

The mean values of $\left\langle X_{\max }\right\rangle$ can be recalculated to the mean values of $\langle\ln A\rangle$ by a simple method of interpolation. The result of such an approach for the points derived from the ADF steepness analysis are shown in Fig. 4. Primary mass composition becomes heavier in the energy range $10^{16}-3 \cdot 10^{16} \mathrm{eV}$ and lighter again in the range $10^{17}-10^{18} \mathrm{eV}$.

\section{Conclusion}

1. Primary CR energy spectrum in the range of $6 \cdot 10^{15}-10^{18} \mathrm{eV}$ has a number of features: the spectrum becomes flatter (the index changes from $\gamma=3.23 \pm 0.01$ to $\gamma=2.98 \pm$ $0.01)$ at $E_{0}=2 \cdot 10^{16} \mathrm{eV}$ and steeper $(\gamma=3.35 \pm 0.11)$ at $E_{0}=3 \cdot 10^{17} \mathrm{eV}$. There is a possibility of more complex description of the second "knee" with a small intermediate increase of the absolute value of the index to $\gamma=3.06 \pm 0.03$ at $E_{0}=8 \cdot 10^{16} \mathrm{eV}$.

2. Tunka-HiSCORE will provide information on the detailed shape of the spectrum at and before the knee after getting more statistics. A preliminary spectrum has been presented.

3. In the energy range of $10^{16}-10^{17} \mathrm{eV}$ observed Tunka spectrum is consistent with the spectra of KASCADE-Grande [5] and Ice-TOP [13].

4. Beyond the energy $10^{17} \mathrm{eV}$ Tunka-133 spectrum is consistent with fluorescent light experiments: Fly's Eye [14], HIRES [15] and hybrid experiment TA [16].

5. The depth of maximum $X_{\max }$ does not contradict to the results obtained by fluorescent light experiments HiRes-MIA [15] and Auger [17].

6. Primary mass composition becomes heavier in the energy range $10^{16}-3 \cdot 10^{16} \mathrm{eV}$ and lighter again in the range $10^{17}-10^{18} \mathrm{eV}$.

This work was supported by the Ministry of Education and Science of the Russian Federation (State Contract 14.V25.31.0010, project 1366, zadanie N 3.889.2014/K), the Russian Foundation for Basic Research (grants 13-02-12095, 13-02-00214, 15-02-05769, 15-02-10005), the Helmholtz Association (grant HRJRG-303) and the Deutsche Forschungsgemeinschaft (grant TL 51-3).

\section{References}

[1] Berezhnev S.F., et al. (Tunka-133 Collaboration), Nucl. Instr. and Methods in Physics Research A 692, 98 (2012)

[2] Budnev N.M., et al., Journal of Instrumentation 9, C09021 (2014)

[3] Tluczykont M., et al., Astropart. Phys. 56, 42 (2014) 
[4] Tluczykont M., et al., 24th ECRS, Kiel (in press) (2014)

[5] Apel W.D., et al. (KASCADE-Gr. Collaboration), Astropart. Phys. 36, 183 (2012)

[6] Aglietta M., et al., EAS-TOP Collaboration, Astropart. Phys. 10, 1 (1999)

[7] Kostunin D., et al., Nucl. Instr. and Methods in Physics Research A 742, 89 (2014)

[8] Prosin V.V., et al., Nucl. Instr. and Methods in Physics Research A 756, 94 (2014)

[9] Garyaka A.P., et al., Journal of Physics G: Nuclear and Particle Physics ID=35, 115201 (2008)

[10] Antony T., et al., Nucl. Instr. and Methods in Physics Research A 513, 490 (2003)

[11] Amenomori M., et al., (Tibet Col.), Astrophys. J. 678, 1165 (2008)

[12] Arqueros F., et al., The HEGRA Collaboration, Astron. Astrophys. 359, 682 (2000)

[13] Aartsen M.G., et al., Phys. Rev. Lett. D 88, 042004 (2013)

[14] Abu-Zayyad T., et al., Phys. Rev. Lett. 84, 4276 (2000)

[15] Sokolsky P. for the HiRes Collaboration, Nuclear Physics B (Proc. Suppl.) 212-213, 74 (2011)

[16] Abu-Zayyad T., et al., Astropart. Phys. 48, 16 (2013)

[17] Schulz A. for the Pierre Auger Collaboration, Proc. 33rd ICRC Rio De Janeiro ID=769, (2013) 